\title{
Das Vergleichen des Wachstumstempos von Hucho hucho L, in den Gewässern Jugoslaviens und Polens
}

\author{
Von
}

Dr. W. J. Kulm at ycki,

Leiter des Laboratoriums für Binnenfischerei am Staatlichen Landwirtschaftlichen Wissenschaftlichen Institut in Bydgoszcz (Polen).

(Mit 2 Textabbildungen).

Ueber das Wachstum des $\mathrm{Huchen} \mathrm{(Hucho} \mathrm{hucho} \mathrm{L.)} \mathrm{in}$ den Gewässern Jugoslaviens hat $\mathrm{M}$ un d a an einem reichen (527 Exemplare) Material, welches aus der Save und Ljubljanica stammte, gearbeitet und seine Ergebnisse in zwei Publikationen (,,Nekaj statističnih podatkov o šuljci lovi v Savi in v Ljubljanici“ - Lovec, Band XII ex 1925, und „Kako uspevajo šulci v naših vodah" - Glasnik Muzejskego Društva za Siovenijo Band IV-VI ex 1926) veröffentlicht. Diese Untersuchungen von Munda sollen als Vergleichsmaterial für die von mir untersuchten Huchen der polnischen Gewässer dienen. Leider ist mein Material nicht so reich an Exemplaren, wie das von $M \mathrm{unda}$ und deshalb muss ich gleich am Anfang ausdrücklich betonen, dass mein Material nicht die gleiche Beweiskraft besitzt, wie das von $\mathrm{Munda}$.

Ueber das Wachstumstempo des Huchen in dem polnischen Fluss Czeremosz habe ich teilweise bereits anlässlich des V. Limnologenkongresses in Budapest im Jahre 1930 berichtet. („Ueber das Vorkommen und die Biologie des Huchen im Czeremoszfluss" - Verhandlungen der InternationaIen Vereinigung für theoretische und angewandte Limnologie - Band V ex 1931). In der Zwischenzeit habe ich weiteres Huchenmaterial aus dem Czeremosz sowie aus dem Prut und Czarna Orawa untersucht. -

An einen Vergleich des Wachstums der Huchen aus den jugoslavischen und polnischen Gewässern kann man erst vom dritten Lebensjahr an herantreten, da $\mathrm{Munda}$ nur ältere Exemplare untersucht hat. -

Das schnelle Wachstum des Huchen aus dem Czeremoszfluss in den ersten drei Jahren habe ich bereits an anderer Stelle 
(op. cit.) ausführlich behandelt. Deshalb sehe ich hier von dieser Schilderung ab. Es muss nur betont werden, dass ein später untersuchter Huchen aus dem Prutfluss (in welchen der Czeremosz mündet) zeigte dasselbe schnelle Wachstum in den ersten Jahren, wie die Czeremoszhuchen.

Die Ergebnisse des Längenzuwachses dieses Pruthuchen nach der Schuppe berechnet, haben im ersten Jahr eine Körperlänge von $14,5 \mathrm{~cm}$ (bei einer Totallänge von circa $18 \mathrm{~cm}$ ), im zweiten $29 \mathrm{~cm}$ (Totallänge circa $32 \mathrm{~cm}$ ), im dritten $45 \mathrm{~cm}$ (Totallänge $49 \mathrm{~cm})$ dargelegt. -

Das schnelle Wachstum des Huchen in den ersten drei Jahren seines Lebens ist auch in Jugoslavien bekannt, wo bei der künstlichen Zucht nach dem ersten Lebensjahr die Länge von 15 bis $20 \mathrm{~cm}$, nach dem zweiten von 30 bis $40 \mathrm{~cm}$ (bei einem Gewicht von $0,5 \mathrm{~kg}$ ) erreicht wurde. Die jugoslavischen Ergebnisse stehen in vollem Einklang mit denen, die mir die Czeremoszhuchen gegeben haben.

Das Wachstumstempo des Czeremoszhuchen vom dritten bis zum sechsten Lebensjahr zeigt die Tabelle 1. -

Tabelle 1.

Wachstumstempo des Czeremoszhuchen vom dritten Jahr an $(n=23)$.

\begin{tabular}{|c|c|c|c|c|}
\hline \multicolumn{2}{|c|}{$\begin{array}{lllll}A & 1 & t & e & r\end{array}$} & \multirow{2}{*}{ Gewicht in $\mathrm{g}$} & \multirow{2}{*}{ Körperlänge in $\mathrm{cm}$} & \multirow{2}{*}{$\mathrm{n}$} \\
\hline Jahre & Monate & & & \\
\hline $\begin{array}{l}3 \\
3 \\
3 \\
3 \\
3 \\
3 \\
4 \\
4 \\
4 \\
4 \\
4 \\
5 \\
5 \\
5 \\
5 \\
6 \\
\end{array}$ & $\begin{array}{l}0 \\
1 \\
1^{1} / 4 \\
1^{1} / 2 \\
3^{1 / 4} / 4 \\
1 \\
1^{1} / 4 \\
1^{1 / 2} / 2 \\
2^{1} / 4 \\
11^{1} / 2 \\
1^{1} / 2 \\
1^{3} / 4 \\
2 \\
11^{1} / 2 \\
11^{1} / 2 \\
\end{array}$ & $\begin{array}{r}1555 \\
1135 \\
975 \\
1050 \\
1400 \\
1500 \\
2100 \\
1600 \\
1640 \\
2700 \\
2950 \\
2520 \\
2700 \\
3650 \\
5500 \\
\end{array}$ & $\begin{array}{l}55 \\
51 \\
48 \\
51 \\
53 \\
52 \\
63 \\
52 \\
59 \\
63 \\
74 \\
68 \\
70 \\
72 \\
79\end{array}$ & $\begin{array}{l}1 \\
3 \\
2 \\
1 \\
2 \\
1 \\
1 \\
1 \\
2 \\
3\end{array}$ \\
\hline
\end{tabular}

In der Tabelle II sind die Ergebnisse der Untersuchungen von Munda zusammengestellt, wobei bemerkt werden muss, dass sowohl die Längen wie die Gewichte nur annähernd angegeben werden konnten, da sie nicht den Zahlentabellen, sondern den Diagrammen entnommen wurden. Tabelle II. - 
Auf Grund dieser beiden Untersuchungen habe ich die folgenden "Wachstumskurven" gezeichnet (Abbildung 1 und Abbildung 2), welche nach dem $\mathrm{Hae} \mathrm{mpels} \mathrm{chen} \mathrm{Vorgehen} \mathrm{konstru-}$ iert wurden, indem auf der Ordinate die Monate, auf der Abszisse die Längen, beziehungsweise Gewichte, der einzelnen von mir untersuchten Fische oder die allgemeinen Ergebnisse der Untersuchungen von $\mathrm{Mund}$ a aufgetragen wurden.

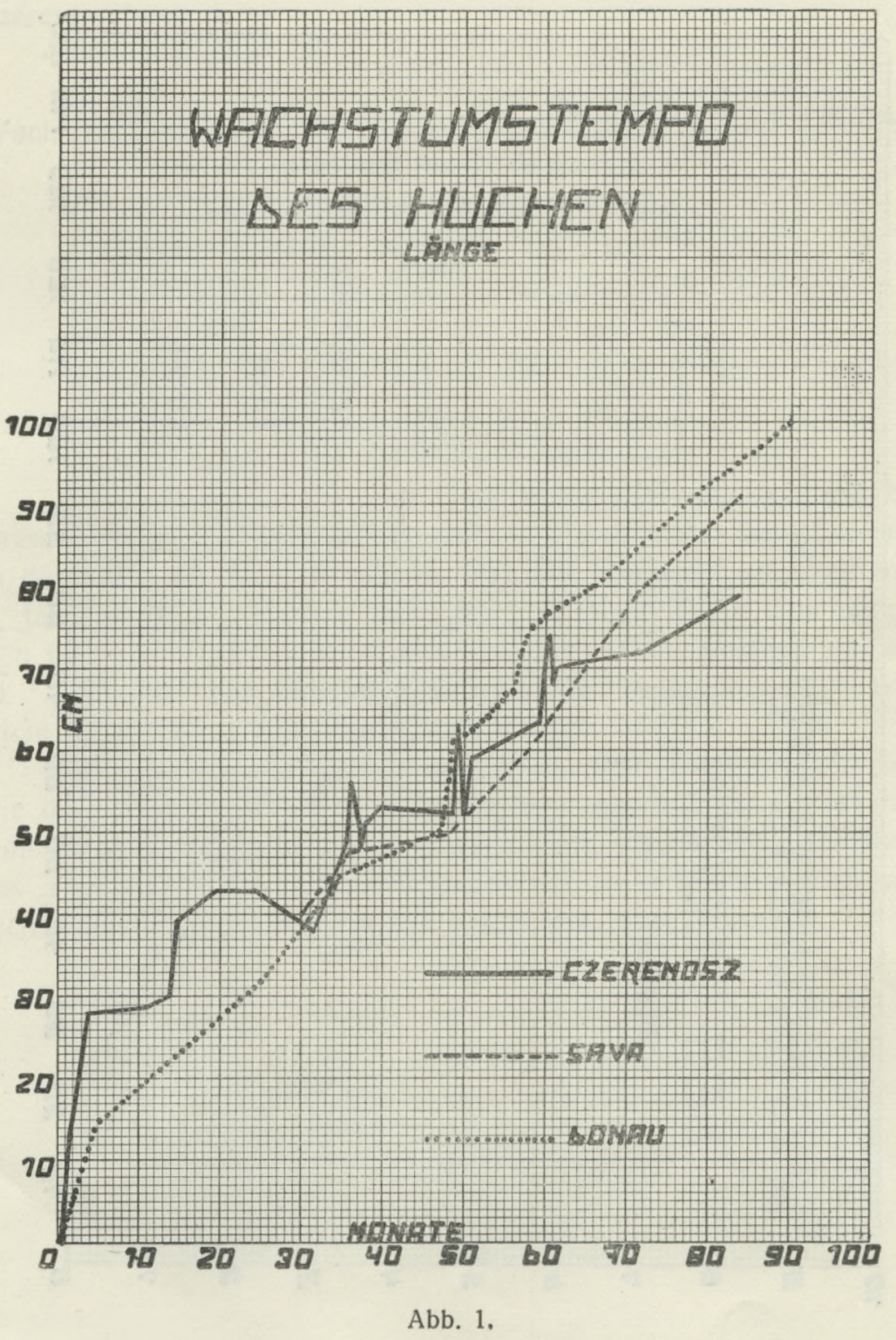


Zum Vergleich habe ich ausserdem in meinen Diagrammen die $\mathrm{H}$ a e m pel-schen Ergebnisse (,Ueber das Wachstum des Huchens, Salmo hucho L./. - Ein Beitrag zur Altersbestimmung der Teleostier". - Internationale Revue der gesamten Hydrobiologie und Hydrographie -- Band III ex 1910-1911) der Untersuchung der Donauhuchen berücksichtigt. -

Die erste Abbildung zeigt das Längenwachstum, die zweite das Gewichtswachstum.

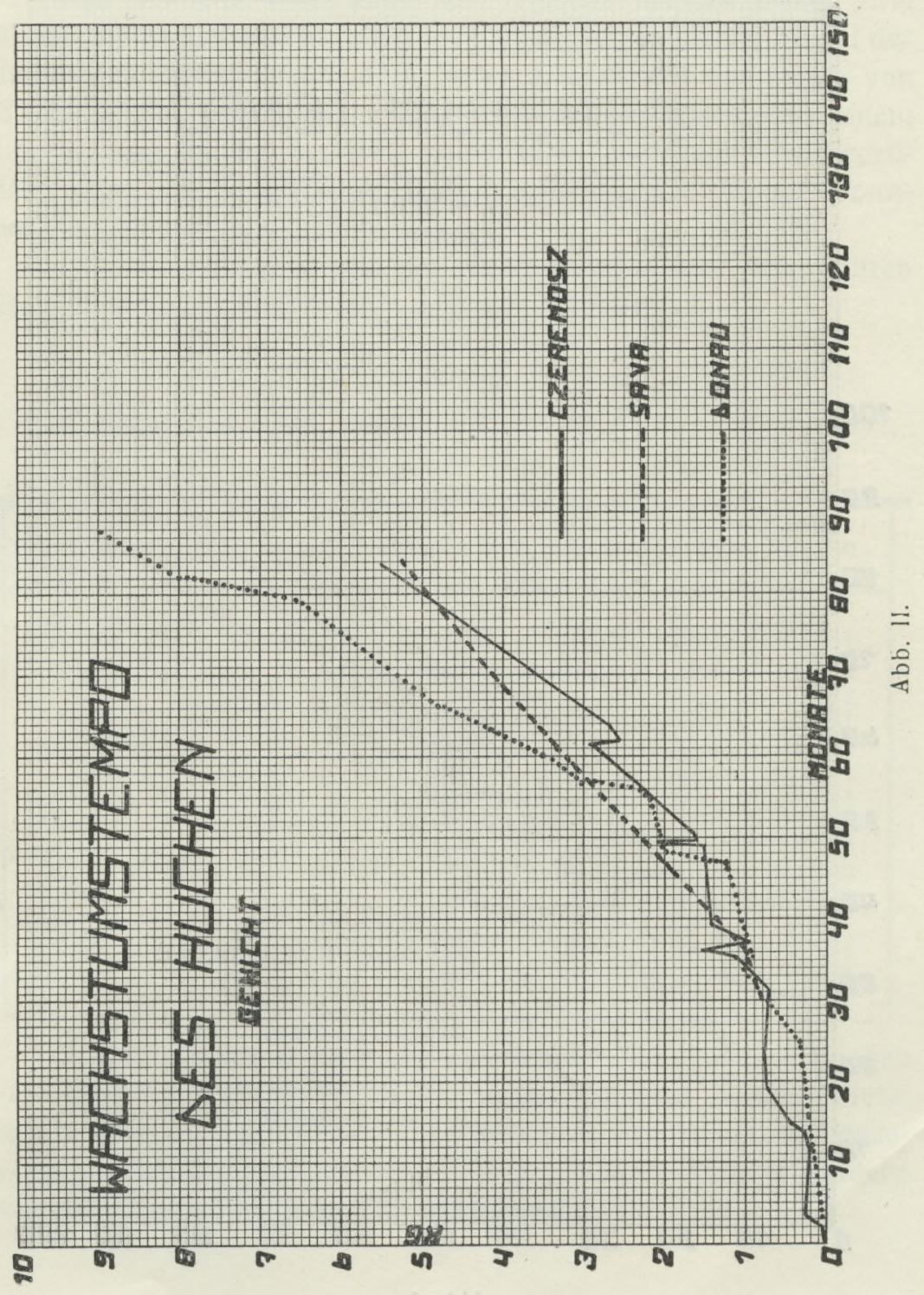


Man sieht deutlich, dass zwischen dem Savahuchen und dem Czeremoszhuchen in dem Wachstum gewisse Unterschiede bestehen. Was die Länge anbetrifft, so wächst der Czeremoszhuchen in der Zeit vom vierten bis zum sechsten Jahr schneller als der Savahuchen, wird dann aber von diesem überholt; trotzdem aber ist das Gewicht des Czeremoszhuchen von vierten Jahr an immer kleiner als beim Savahuchen. Wir sehen daraus, dass der Savahuchen eine mehr fleischansetzende Rasse darstellt, als der Czeremoszhuchen. -

Tabelle II.

Wachstumstempo des Savahuchen vom dritten bis zum siebenten Lebensjahr (nach Munda).

\begin{tabular}{|c|c|c|}
\hline $\begin{array}{c}\text { Alter in } \\
\text { Jahren }\end{array}$ & $\begin{array}{c}\text { Gewicht } \\
\text { in kg }\end{array}$ & Länge in cm \\
\hline 3 & 1 & 48 \\
4 & 2 & 60 \\
5 & 3,2 & 73 \\
6 & 4,4 & 80 \\
7 & 5,2 & 81 \\
\hline
\end{tabular}

Die Untersuchung zweier Huchenexemplare aus dem Czarna Orawafluss zeigte, dass der Huchen in diesem Fluss schneller, als in der Sava zu wachsen scheint. Das eine Exemplar im Alter von 3 Jahren $11 \%$ Monate (also circa 4 Jahre) zeigte eine Länge von $65 \mathrm{~cm}$, bei $2800 \mathrm{~g}$ Körpergewicht, das andere, welches 5 Jahre $11^{1}$. Monate (also circa 6 Jahre) alt war, besass bei einem Gewicht von $7150 \mathrm{~g}$ eine Länge von $89 \mathrm{~cm}$. -

Die Untersuchungen von $\mathrm{Mund}$, von $\mathrm{Ha}$ e mpel, sowie die meinigen bezeugen deutlich, dass die einzelnen Flüsse des Donaustromgebietes bestimmt gewisse Unterschiede im Wachstum des Huchen zeigen. Diese Unterschiede zu erforschen wird ein recht interressantes Kapitel der Fischereibiologie sein. - 


